\title{
Stark effect in parabolic quantum dot
}

\author{
S. JAZIRI, G. BASTARD ${ }^{*}$ and R. BENNACEUR ${ }^{* *}$
}

Département de Physique de l'E.N.S. de Bizerte, 7021 Jarzouna Bizerte, Tunisia

* Laboratoire de Physique de la Matière Condensée de l'E.N.S. de Paris, 24 rue Lhomond, 75231 Paris cedex 05, France

** Département de Physique de la Faculté des Sciences de Tunis, Campus Universitaire Le Belvédere Tunis, Tunisia

\begin{abstract}
We theoretically investigate the optical properties of the exciton confined in parabolic quantum-dot, with and without electric field, by means of perturbative-variational method. The quantum-dot size enhances the $1 \mathrm{~S}$ eigenvalue and oscillator strength. In smaller dot the relative extension of the exciton wave function is equal to the size of the dot. The 1S exciton binding energy is found to be almost 2-3 times that in the quantum-well of the same thickness. In the presence of an external electric field, we calculate the quantum-confined Stark effect . The energy split is found about the same as in quantum-well with the same size .The wider quantum-dot has a larger Stark shift. We also analyse the special case of high electric field. In this case the Coulombic interaction can be approximated by parabolic potential.
\end{abstract}

\section{1-INTRODUCTION}

In recent years excitons states in quantum dots have been studied in a number of papers [1-4] and have been observed by photoluminescence experiments [56 ]. The study of electronic states in quantum dots depends on either the confining potential and the interacting force between the particles. Following the" Generalized Kohn's Theorem "; theoretical studies show that the confined potential for electrons [7-9] and holes [2,9] in quantum dots is nearly parabolic, so the center-of-mass motion can be solved exactly. The effect of an electrostatic field on the electron-hole states and on the confined excitonic states is referred to the quantum confined Stark effect (Q.C.S.E.) has received intensive discussions in quantum well structures [12,15] and few studies in quantum wire and in quantum dot systems [13]. In this work, using a simple and efficient approximation, we propose to study the exciton properties in a parabolic quantum dot structure with and without the presence of an electrostatic field. In sect.2-, we present the formalism of the perturbativevariational method [14]. We investigate the Stark shifts. We also analyse the special case of high electric field. The results for the exciton ground state properties in parabolic quantum dot, and the energy level split under an electric field, are presented and discussed in sect.3- .

2- THEORY

Within the effective-mass approximation and neglecting the band-structure effects, the Hamiltonian of an exciton in a parabolic quantum dot with the 
same quantization energy $k \Omega$ ( for the electron and hole)[2], and subjected to an external electric field, can be expressed as :

$$
H=\frac{P_{e}^{2}}{2 m_{e}}+\frac{1}{2} m_{e} \Omega^{2} r_{e}^{2}+\frac{P_{h}^{2}}{2 m_{h}}+\frac{1}{2} m_{h} \Omega^{2} r_{h}^{2}-\frac{e^{2}}{\varepsilon r}+e F z_{e}-e F z_{h}
$$

where $m_{e}\left(m_{h}\right)$ are the single-particle Hamiltonian and the effective-mass for the electron (hole), $\varepsilon$ is the background dielectric constant . Using the relative coordinate $r=\left(r_{e}-r_{h}\right)$ and the corresponding momenta $p$ with reduced mass $\mu=m_{e} m_{h} / M$ and center-of-mass coordinate $R=m_{e} r_{e}+m_{h} r_{h} / M$ and the corresponding momenta $P$ with the total-mass $M=m_{e}+m_{h}$, the Hamiltonian $H$ is represented as :

$H=\frac{P^{2}}{2 M}+\frac{1}{2} M \Omega^{2} R^{2}+\frac{p^{2}}{2 \mu}+\frac{1}{2} \mu \Omega^{2} r^{2}-\frac{e^{2}}{\varepsilon r}+e F z$

In Eq. (2) the part which depends only on the center-of-mass coordinate corresponds to the Hamiltonian of a well-known three dimensional harmonic oscillator. The exciton properties is essentially determined by the relative Hamiltonian. As under the influence of the electric field the potential energy is z-axial symmetric,we use conventional cylindric coordinates. The field term added to the $z$-direction confinement describes a displaced harmonic oscillator centred in $-z_{0}=-\frac{e F}{\mu \Omega^{2}}$ with the frequency $\Omega_{r}$ inferior to $\Omega$. In order to solve the Hamiltonian $\mathrm{H}_{r}$, we introduce an interaction potential which obeys to the Hooke's force with the parameter $\lambda$ by adding and substracting the potential $V(r)=\lambda\left(\frac{1}{2} \mu \Omega^{2} r^{2}-K \Omega\right)$ to be able to split the Hamiltonian $H_{r}$ into two terms, with the one term being exactly solvable while the other can be treated as a perturbation. This potential is similar to the interaction potential between electron-electron used by Johson et al [11]. This approximation is not correct for all electron-hole separation but the interaction parameter $\lambda$ can be adjusted to give the best fit of the true interaction which is the Coulomb interacion, and for the dominant range of separation $r$. The attraction potential $V(r)$ must have negative value with positive $\lambda$, this yields a reasonable fit to the exact interaction for electron-hole separation $r<\sqrt{2} \quad R_{0}$ ' where $R_{0}$ is the quantum dot radius defined by $R_{0}=\sqrt{\frac{K}{\mu \Omega}}$ We determine the best choice of $\lambda$ as the one wich ensures the fasted convergence of the perturbation series. We rewrite $H_{r}$ as :

$$
\mathrm{H}_{\mathrm{r}}=\mathrm{H}_{0}+\mathrm{H}_{1}
$$

in which : $H_{0}=\frac{p^{2}}{2 \mu}+\frac{1+\lambda}{2} \mu \Omega^{2} r^{2}-\lambda \nvdash \Omega-\frac{e^{2} F^{2}}{2 \mu \Omega^{2}}$

$$
H_{1}=-\frac{e^{2}}{\varepsilon \sqrt{\rho^{2}+\left(z-z_{0}\right)^{2}}}-\lambda\left(\frac{1}{2} \mu \Omega^{2} r^{2}-K \Omega\right)
$$


$H_{1}$ is taken as a perturbation by choosing an applicable value of $\lambda$ in the quantum dot ; and $\mathrm{H}_{0}$ as the unperturbed part of the Hamiltonian $\mathrm{H}_{\mathrm{r}}$. The total energy corresponding to exciton ground state is obtained as :

$E_{\mathrm{T}}=\frac{3}{2} k \Omega+\frac{3}{2} k \Omega_{\mathrm{r}}-\lambda k \Omega-\frac{e^{2} F^{2}}{2 \mu \Omega^{2}} \quad$ where $\Omega_{\mathrm{r}}=\Omega \sqrt{1+\lambda}$

The parameter $\lambda$ may be determined[4,14] by: $H_{1}(\lambda)=\left\langle\psi(r, \lambda)\left|H_{1}\right| \psi(r, \lambda)\right\rangle=0$

with $\psi(r, \lambda)$ is the exciton enveloppe wave function. The field-induced energy shifts can be expressed as: $\Delta E=E_{T}(F=0)-E_{T}(F), E(F=0)$ is the corresponding energy in the zero-field state.We calculate the exciton binding energy defined by $E_{B}=E_{e}+E_{h}-E_{T}$, where $E_{e}, E_{h}$ are the energies corresponding to the singleparticle Hamiltonian. The extension of the exciton relative motion is calculated by the expectation value in the ground state of $r$. We also calculate the oscillator strength of the exciton ground state in the dot normalised to that of a free exciton in a bulk material with volumeV $=4 / 3 \pi R_{0}^{3}$ which given by

[4]: $f / f_{e x}=\frac{E_{e x}}{E} \frac{\pi a^{* 3}}{V}\left|\int \chi(R, 0, \lambda) d^{3} R\right|^{2}$

where $E_{e x}=E_{G}-R^{*}$ and $E=E_{G}+E_{T}$ are the exciton energies in the bulk and in the quantum dot respectively, and $a^{*}$ is the effective Bohr radius, $R^{*}$ is the effective Rydberg energy and $E_{G}$ is the gap energy and $\chi(R, r, \lambda)$ is the total exciton enveloppe wave function

Effect of strong electric field

Due to the confinement effects the enhancement of the exciton binding energy in quantum dot is found to be significantly large. It is interesting to investigate the particular large field effects on the confined exciton in parabolic quantum dot. One possible approach is the perturbative-variational method as mentioned in the last subsection. A better way is to analyse in this case the expanding of the Coulomb interaction . Large electric field induces a spatial shift of the particles along the field direction, leading to a reduction of the Coulomb interaction which can be approximated by:

$V(\rho, z)=-\frac{e^{2}}{\varepsilon}\left[\frac{1}{z_{0}}-\frac{\rho^{2}}{2 z_{0}^{3}}+\frac{z}{z_{0}^{2}}-\frac{z^{2}}{z_{0}^{3}}-\frac{3 z \rho^{2}}{2 z_{0}^{4}}+\frac{z^{3}}{z_{0}^{4}}+\ldots.\right]$

We only consider the first and the second order of the expansion in the relative Hamiltonian $H_{r}$. The longitudinal relative motion of the exciton is a linear harmonic oscillator pushed on the left by quantity $\left(z_{0}-z_{1}\right)$, and displayed with the frequency $\Omega$ // inferior to $\Omega$, due to combined effects of the electric field and Coulombic potential. The transverse relative-motion is represented by a flater harmonic oscillator with the frequency $\Omega_{\perp}$ superior to $\Omega$.The energy of the exciton in the ground state is given by :

$\mathrm{E}_{\mathrm{T}}(\mathrm{F})=\frac{3}{2} k \Omega+k \Omega_{\perp}+\frac{1}{2} \hbar \Omega_{/ /}-\lambda K \Omega-\frac{\mathrm{e}^{2} \mathrm{~F}^{2}}{2 \mu \Omega^{2}}-\frac{e^{2}}{\varepsilon z_{0}}-\frac{1}{2} \mu \Omega_{/ /}{ }^{2} \mathrm{z}_{1}{ }^{2}$

with $\Omega_{/ /}=\Omega \sqrt{1-2 \xi / z_{0}}, \Omega_{\perp}=\Omega \sqrt{1+\xi / z_{0}}$ and $z_{1}=\frac{\xi}{1-2 \xi / z_{0}}$ where $\xi=\frac{e^{2}}{\varepsilon \mu z_{0}{ }^{2} \Omega^{2}}$

Obviously the expansion of the Coulomb interaction is not correct for all field strength. In order for the model to be applicable, we must determine a 
condition which makes the approximation of the Coulomb attraction physically reasonable. The longitudinal relative coordinate $z_{0}$ should be greater than the relative position coordinates particulary than the mean value of the displaced harmonic oscillator in the $z$-direction which is equal to $z_{1}$. Then the validity criterion of this method is found for $z_{1} \ll z_{0}$ leading the following condition :

$$
\left(\frac{\mathrm{eFa}^{*}}{2 \mathrm{R}^{*}}\right)^{3}\left(\frac{\mathrm{R}_{\mathrm{o}}}{\mathrm{a}^{*}}\right)^{8}>>1
$$

This approximation is valid for large enough electric field and small dots or for weak electric field and wide dots .

\section{RESULTS}

As a numerical example, we choose a GaAs semiconductor quantum dot, with the parameter values: dielectric constant $\varepsilon=13.1$, effectivemasses for the electron (e), heavy hole (hh) and the light hole $(\mathrm{lh}), \mathrm{m}_{\mathrm{e}}=0.067 \mathrm{~m}_{\mathrm{o}}$, $m_{h h}=0.377 m_{0}$ and $m_{1 h}=0.09 m_{0}\left(m_{\circ} E(m e V)\right.$
is the free-electron mass ). In the absence of the electric field we plot in fig. 1 the ground state energy and the binding energy. For narrower dots, the effect of the parabolic confinement is more pronouced, leading to increasing exciton energies. We can see the decreasing of the binding energy of the heavy -hole exciton confined in quantum dot with the increasing dot size, and is found to be almost $2-3$ times that of the one confined in quantum well [12] with the same size. The enhancement of the energy of the light-hole exciton is larger than that of heavy-hole for the same quantum dot radius, as expected from the lighter carrier mass, the quantum confinement effect appears more for (e-lh) than (e-hh) exciton.

Fig. 2 shows the electron-hole separation of the heavy- and light-hole exciton in the ground state, for narrower dots, the curve is linear and the normalized electron-hole position $\langle r\rangle / R_{0}$ approaches to the result deduced by $[2,3]$ equal to the unity . 
The electron-hole separationbecomes insensitive to the wide quantum dots and converge to the bulk value separation. The size dependence of the normalized oscillator strength (fig.2) is determined essentially by the integral in eq.(6), while the energy dependent term displays negligible variations [2-4]. As the quantum dot size is increased the envelope function becomes more and more flat, the integral term decreases rapidely and converges towards constant . This point is already been noticed by $[2-4$. This result should be general independent of the confining and interaction potentials.

The electric field effects on exciton in GaAs parabolic quantum dot are plotted in Fig 3,4. In fig. 3 we plot the calculated heavy-hole exciton resonance energy shift $\Delta E$ versus the electric field strength for several quantum-dot radius.

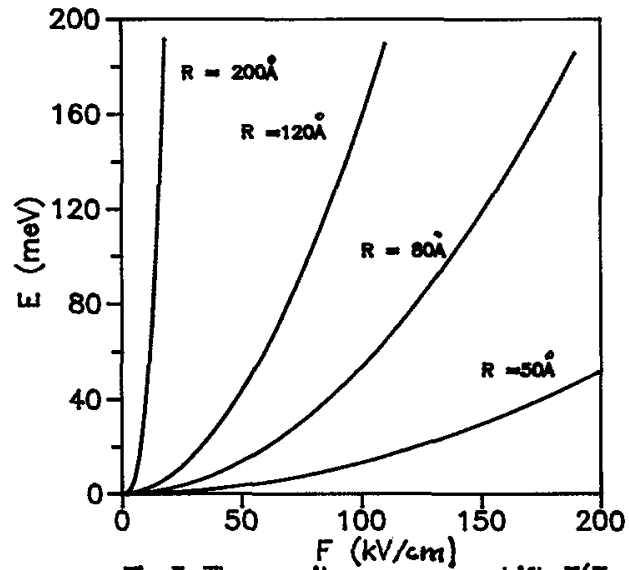

Fig.3 The exciton energy shift $E(F=0)-E(F)$ versus electric field for different quantum dots.

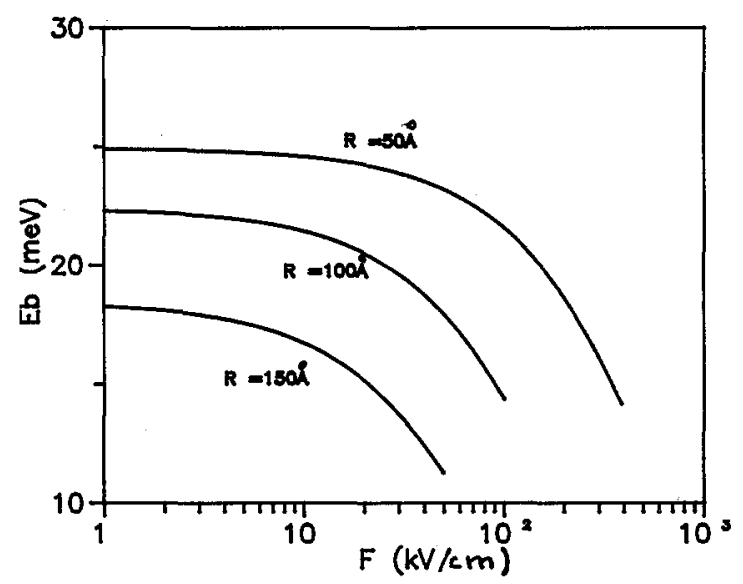

Fig.4The exciton binding energy as a function of electric field for different quantum dots.

The split variation of the exciton energy is found about the same as in parabolic quantum-well [13] and is a few larger than the excitonic shift in the square quantum-well case with the same thickness [12]. The 1S binding energy of the heavy-hole exciton is shown in fig. 4 as a function of an applied electric field for three different quantum-dot radius. Finally, we examine the case where the Coulombic potential is approximated by an interaction potential which is composed essentially by quadratic terms. We investigate the energy states of the exciton in parabolic quantum-dot subjected to an applied electric field when the condition (9) is satisfied : high field strength for narrower quantum-dot and relatively weak field strength for wider quantum-dot. We have compared these energies with those determined by the variationalperturbative method. The corresponding accuracy is of the order of $1 \%$. It should be noted that the actual method is much simpler but cannot enviseaged for any strength electric field and/nor any quantum dot size.

\section{REFERENCES}

[1]- Y.Z. Hu , M.Lindberg and S.W.Koch ,Phy.Rev. B 42 ,1713 (1990).G.W. Bryant, Phys. Rev.B 37,8763 (1988) andT. Takagahara, Surf. Sci. 267 , 310 (1992).

[2]- Weiming Que, Solid Stat. Comm. , 81, 721 (1992).

[3]- Y.Kayanuma, Phys.Rev B 44,13085 (1991) . 
[4]-S.Jaziri,G.Bastard and R.Bennaceur,Semicond.Sci.andTechnol.(to be published) [5]- K. Kash ,A.Scherer,J.M. Worlock, H.G. Craighead and M. C. Tamargo,Appl. Phys. Lett.49,1043 (1986).

[6] M. A. Reed, R.T. Bate, K. Bradshaw, W.M. Duncan , W. R.Frensley, J.W. Lee and H.D. Shih , J. Vac. Sci Technol. B4 ,358 (1986).

[7]- L. Brey, N.F. Johnson and B.I Halperin , Phys. Rev. B 40,10647 (1989).

[8]- P.A. Maksym and T. Chakraborty , Phys. Rev. Lett. 65 ,108 (1990)

[9]- F.M.Peeters , Phys. Rev. B 42,1486 (1990).

[10]-V. Halonen, T. Chakraborty and P. Pietilainen Phys. Rev. B 45,5986 (1992).

[11]- N.F. Johnson and M.C. Payne, Phys. Rev.Lett , 67,1157 (1991).

[12]- G. Bastard ,"Wave Mechanics Applied to Semiconductor heterostructures ", Les Editions de Physique, Les Ulis (1988) ;D.A.B. Miller, D.S. Chemla, T.C. Damen, A.C. Gossard, W. Wiegmann, T.H. Wood and C.A. Burrus , Phys. Rev. B 32,1043 (1985).- G. Bastard and J.Brum, IEEE J. Quantum Electron. Q-E 22,1625 (1986).

[13]-Y. Chiba and S.Ohnishi, Phy.Rev.B, 38, 12988 ( 1988).

[14]-Y.C. Lee ,W.N. Mei and K.C.Liu, J.Phys. C15, L469 (1982).

[15]- Chen and T.G. Andersson, Semicond. Sci. Technol. 7828 (1992). 